\title{
Star Formation History and Chemical Evolution of Resolved Galaxies: a New Model
}

\author{
Myung Gyoon Lee ${ }^{1}$ and In-Soo Yuk ${ }^{2}$ \\ ${ }^{1}$ Astronomy Program, School of Physics and Astronomy, Seoul National University, Seoul \\ 151-742, Korea \\ email: mglee@astrog.snu.ac.kr \\ ${ }^{2}$ Korea Astronomy and Space Science Institute, 61-1 Hwaam-dong, \\ Yuseong-gu, Daejeon 305-348, Korea \\ email: yukis@kasi.re.kr
}

\begin{abstract}
We present a new numerical model of star formation history and chemical evolution using the color-magnitude diagrams of resolved stars in galaxies. Our model differs from previous models in that it generates the simulated color-magnitude diagrams with the metal enrichment law calculated from the galactic chemical evolution model. We present a case of applying our model to a resolved galaxy in the Local Group: the Sextans dwarf spheroidal galaxy. It is found the the primary origin of the radial gradient of stellar population in this galaxy is star formation followed by metallicity.
\end{abstract}

Keywords. galaxies:evolution, galaxies:dwarf, galaxies:Local Group, galaxies:individual (Sextans dwarf spheroidal galaxy), stars: formation

\section{Introduction}

Deriving star formation history and chemical evolution of galaxies is a crucial key to understanding the formation and evolution of galaxies. Nearby resolved galaxies where we can investigate details of various stellar populations are ideal targets in this regard. To date there have been good progress in two lines of modelling: models of star formation history on one hand, and models of chemical evolution on another hand (see Dolphin (2002) and references therein). However, they were developed in most cases independently from each other. In this study we present a new numerical model of star formation history and chemical evolution using the color-magnitude diagrams of resolved stars in galaxies. Our model differs from previous models in that it generates the simulated color-magnitude diagrams with the metal enrichment law calculated from the galactic chemical evolution model. Hence our model is free from the problem of age-metallicity degeneracy, and provides us with the information of chemical enrichment history as well as star formation history of galaxies.

\section{The Model}

The goal of our model is to derive simultaneously the star formation history and chemical evolution of galaxies from observational color-magnitude diagrams (CMDs). The methodology is to produce a set of simulated CMDs for given range of star formation history and then to compare the simulated CMDs with the observational CMD, finding one that fits best the observational CMD. The best-fit time variations of star 


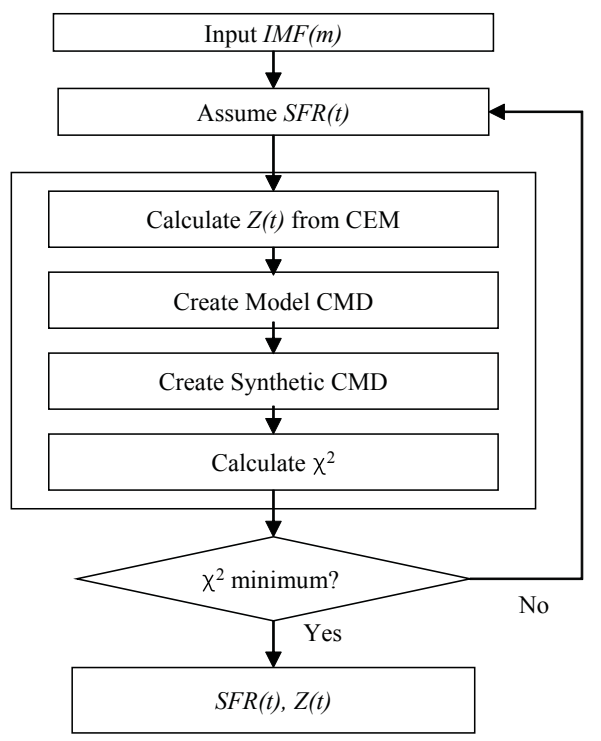

Figure 1. A flow chart for deriving the star formation rate $S F R(t)$ and metallicity $Z(t)$ from the observational CMDs.

formation rate and metallicity are determined by minimizing the goodness-of-fit parameter $\chi^{2}$ calculated from the comparison of observational CMDs and simulated CMDs.

Figure 1 displays a flow chart of our model for deriving the star formation rate and metallicity as a function of time. The distance modulus $(m-M)_{o}$ and the reddening $E(B-V)$ are adopted as input parameters, and a simple functional form for the initial mass function $\operatorname{IMF}(\mathrm{m})$ is used in our model. First, we assume a star formation rate $S F R(t)$ in the beginning, and then calculate the metallicity $Z(t)$ using galactic chemical evolution model (CEM) with given $S F R(t)$ and $I M F(m)$. The next step is to construct a model CMD using the theoretical isochrones, and then convert it to the synthetic CMD considering photometric errors and completeness. The last step is to calculate the goodness-of-fit parameter, $\chi^{2}$ from the comparison of observational CMD and the synthetic CMD, checking whether the value of $\chi^{2}$ is minimum or not. If the value of $\chi^{2}$ is not minimum, then we change the $S F R(t)$ and repeat the above procedure until the minimum $\chi^{2}$ is found. The best-fit star formation history is found from the smallest $\chi^{2}$. The most distinguishable difference between other models and our model is the way to determine metallicity. While other models assume the metallicity a priori, our model calculates it using the galactic chemical evolution model (for the calculation of chemical evolution we followed mainly Timmes, Woosley \& Weaver (1995)).

\section{Testing the Model}

We have tested our model for the artificial galaxies having different star formation histories and for the Local Group dwarf irregular galaxy IC 1613. Our model successfully reproduces the star formation history and chemical evolution of the artificial galaxies. The star formation and metal enrichment histories of IC 1613 derived in this study are consistent with those in previous studies (e.g., Skillman et al. (2003)) as shown in Figure 2 . 


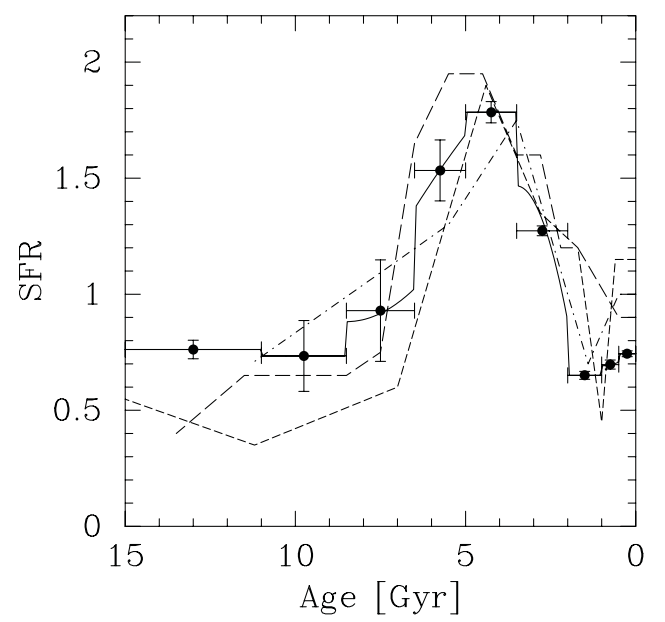

Figure 2. The best-fit star formation history of IC 1613 derived in this study (filled circle with error bars) in comparison with the models of Tolstoy (long dashed line), Cole (dashed line), and Dolphin (dash-dot line) in Skillman et al. (2003). The star formation history is averaged over the time bin. The horizontal bar represents the size of a time bin, and the vertical bar indicates the errors of $1 \sigma$ level.

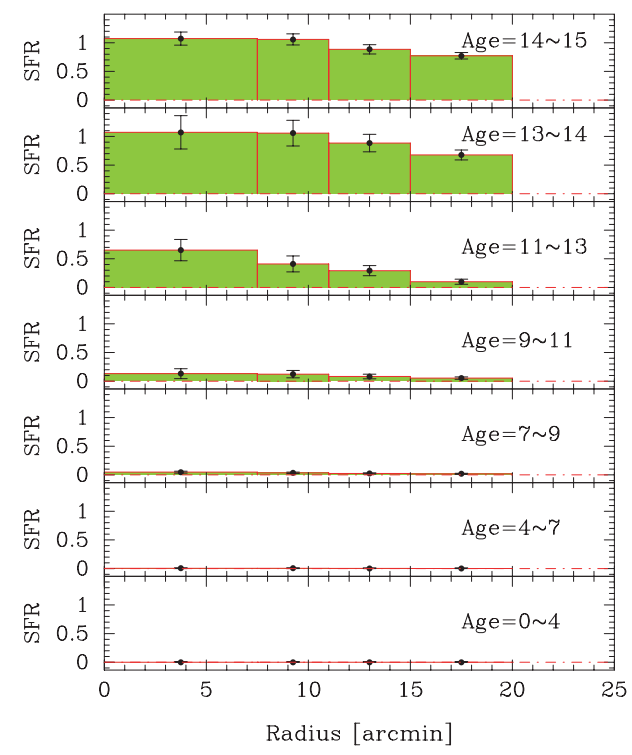

Figure 3. Radial distribution of the star formation history of the Sextans dSph. The vertical bars indicate the symmetric errors of $1 \sigma$ level.

\section{Application: the Sextans Dwarf Spheroidal Galaxy}

We applied our model to one of nearest resolved galaxy in the Local Group: the Sextans dwarf spheroidal galaxy (dSph). It is known that there is a significant radial gradient of stellar population in this galaxy (Lee et al. (2003)) We have derived the star formation history and chemical evolution from the $V I$ photometry of stars in the $42^{\prime} \times 28^{\prime}$ field of this galaxy given by Lee et al. (2003). We considered two cases in our application of the model: excluding the blue stragglers and including the blue stragglers in which case blue 


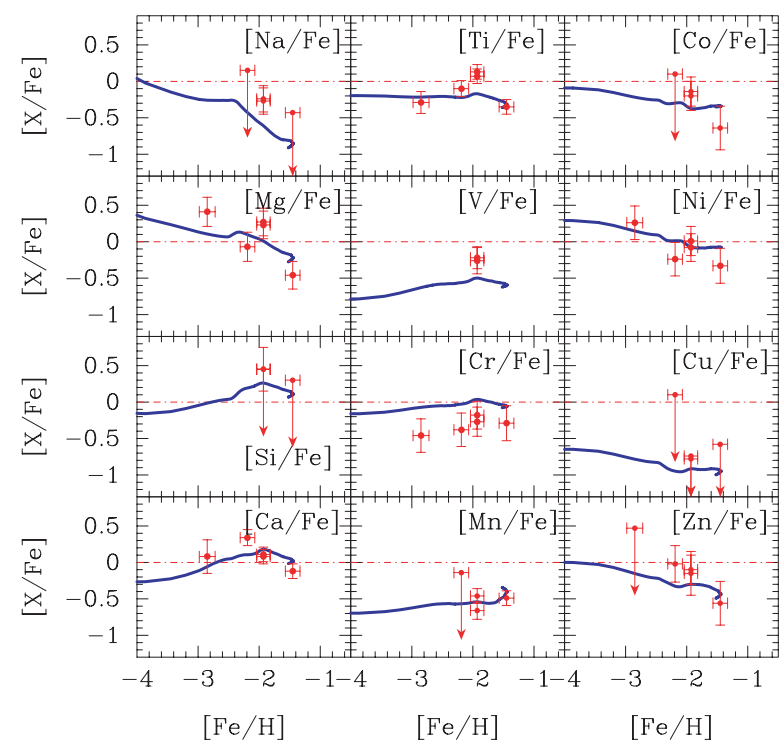

Figure 4. Abundance ratios for the elements in the Sextans dSph. Filled circles with error bars represent the observed data given by Shetrone, Côté \& Sargent (2001), and solid lines represent the calculated values in this study.

stragglers are treated as younger main sequence stars. Here we describe only the first case. We adopted a closed-box model for chemical evolution. Figure 3 displays the radial variations of the star formation history of the Sextans dSph. It is found that about $65 \%$ of the stars were formed before 13 Gyr ago, about $25 \%$ of stars were formed between 13 Gyr ago and 11 Gyr ago, and a small fraction of stars were continuously formed until 7 Gyr ago. Star formation was more active in the inner region than in the outer region of the galaxy. Metallicity of the stars was increased upto $[\mathrm{Fe} / \mathrm{H}]=-1.6$ (in the central region) and -1.8 (in the outer region) by $14 \mathrm{Gyr}$ ago, and remains almost constant since then. Figure 4 show abundance ratios for the elements in the Sextans dSph. The abundance ratios of most elements derived in this study are in good agreements with the observed results based on the high resolution spectroscopy (Shertone et al. (2001)), but those of some elements are not. There results show that the primary origin for the well-known radial gradient of stellar population in this galaxy is probably be star formation, followed by metallicity.

\section{Acknowledgements}

This work is in part is supported in part by the grant, (R01-2004-000-10490-0) from the Basic Research Program of the Korea Science and Engineering Foundation

\section{References}

Dolphin, A. E. 2002, MNRAS, 332, 91

Lee, M. G., Park, H. S., Park, J. H., Sohn, Y. J., Oh, S. J., Yuk, I. S., Rey, S. C., Lee, S. G, Lee, Y. W., Kim, H. I., Han, W, Park, W. J, Lee, J. H, Jeon, Y. B., \& Kim, S. C. 2003, AJ, 126,2840

Skillman, E. D., Tolstoy, E., Cole, A. A., Dolphin, A. E., Saha, A., Gallagher, J. S., DohmPalmer, R. C., \& Mateo, M. 2003, ApJ, 596, 253

Shetrone, M. D., Côté, P., \& Sargent, W. L. W. 2001, ApJ, 548, 592 


\section{Discussion}

GEISLER: What is your prediction for $[\alpha / \mathrm{Fe}]$ at the present day metallicity in IC1613?.

LEE: I do not have it with me now but will send it to you later.

R. DE Jong: You find extended SFH for Sextans. However, did you do the test of the previous speaker (Aparicio). Could you recover a single burst of SF, when using a mismatch in evolutionary tracks for creating the artificial CMD and for recovering the SFH?.

LEE: We have used only one set of stellar evolution tracks (Padova library), and did not have a chance to check the mismatch problem.

Bland-Hawthorn: Particularly with regard to considering heavy elements. This is very interesting work. I would encourage you now to determine what kinds of data can advance this field. Do you need high resolution spectroscopy or can you use intermediate or narrow band imaging?.

LEE: .As long as the information of the chemical abundances (either each or a group of several elements) is available, either high resolution spectroscopy or intermediate or narrow-band imaging is useful. However, abundance ratios of stars in these galaxies are crucial to derive the details of chemical evolution. So we encourage astronomers to obtain high resolution spectra of these stars.

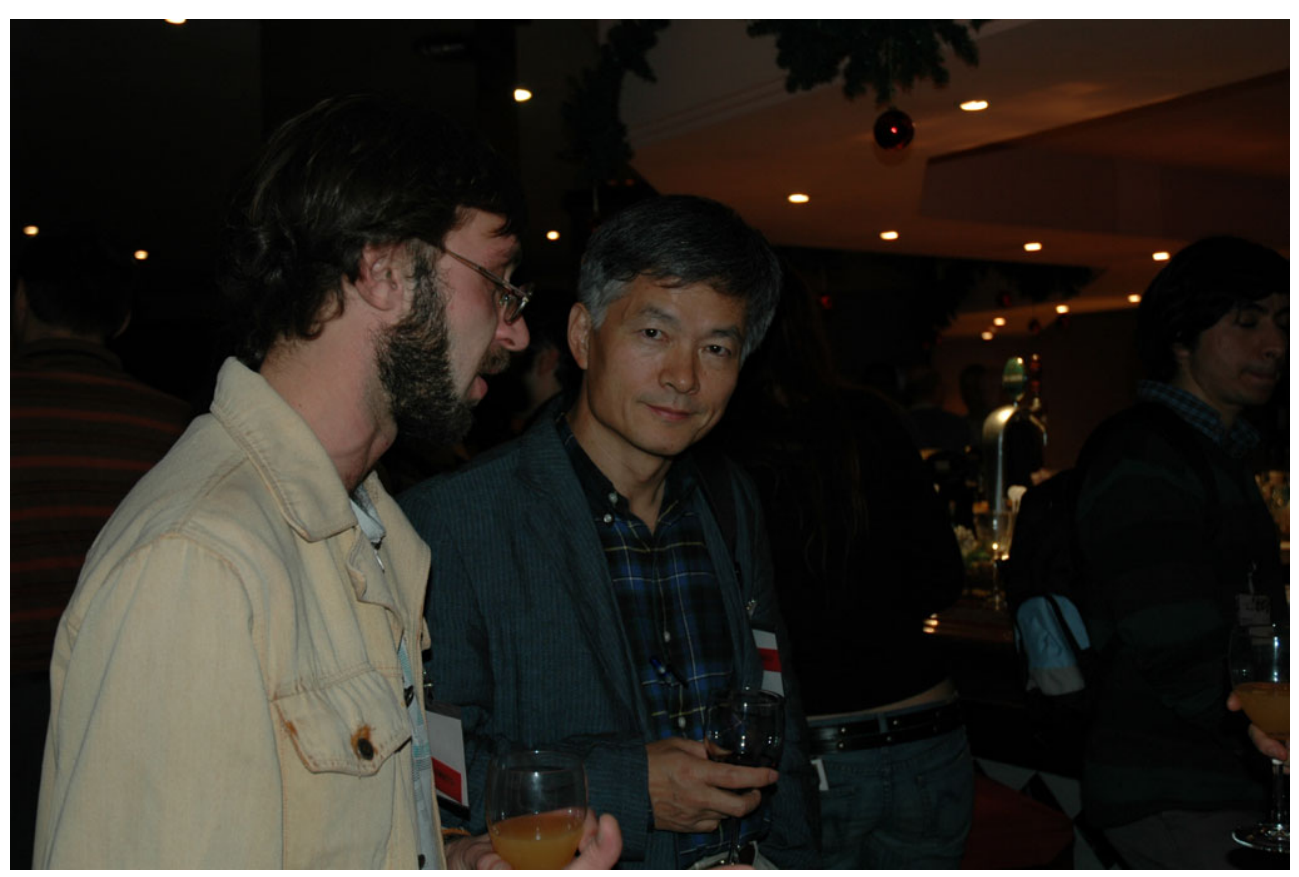

The author (middle) with Alexander Gusev and Mario Soto (right). 